\title{
Correction to: Ophiostomatoid fungi associated with Ips subelongatus, including eight new species from northeastern China
}

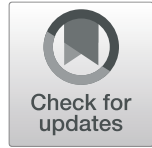

Zheng Wang ${ }^{1}$, Ya Liu' ${ }^{1}$, Huimin Wang ${ }^{1}$, Xianjing Meng ${ }^{1}$, Xuewei Liu ${ }^{1,2}$, Cony Decock ${ }^{3}$, Xingyao Zhang ${ }^{1}$ and Quan Lu'

\section{Correction to: IMA Fungus (2020) 11:3 \\ https://doi.org/10.1186/s43008-019-0025-3}

Following publication of the original article (Wang et al. 2020), the authors reported that Table 1 contained some errors in the GenBank number of BT gene. The correct Table 1 is presented below.

\footnotetext{
Author details

${ }^{1}$ Key Laboratory of Forest Protection, National Forestry and Grassland Administration; Research Institute of Forest Ecology, Environment and Protection, Chinese Academy of Forestry, Beijing 100091, China. ${ }^{2}$ Wuqing Forestry Bureau, Tianjin 301700, China. ${ }^{3}$ Mycothèque de I'Université Catholique de Louvain (MUCL), Earth and Life Institute, Microbiology, B-1348 Louvain-la-Neuve, Belgium.
}

Published online: 20 April 2020

\section{Reference}

Wang Z et al (2020) Ophiostomatoid fungi associated with Ips subelongatus, including eight new species from northeastern China. IMA Fungus 11:3. https://doi.org/10.1186/s43008-019-0025-3

\section{*Correspondence: luquan@caf.ac.cn}

'Key Laboratory of Forest Protection, National Forestry and Grassland Administration; Research Institute of Forest Ecology, Environment and Protection, Chinese Academy of Forestry, Beijing 100091, China

Full list of author information is available at the end of the article

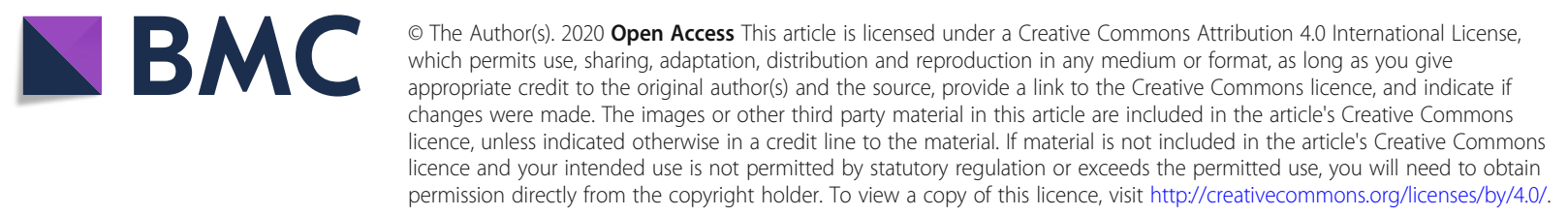


Table. 1 Strains of ophiostomatoid fungi sequenced and used for morphological and phylogenetic analysis in this study

\begin{tabular}{|c|c|c|c|c|c|c|c|c|}
\hline \multirow[t]{2}{*}{ Taxon } & \multirow[t]{2}{*}{ Species $^{1}$} & \multirow[t]{2}{*}{ Strain no. ${ }^{2.3 .4}$} & \multirow[t]{2}{*}{ Host } & \multirow[t]{2}{*}{ Locality } & \multicolumn{4}{|c|}{ GenBank number $^{5}$} \\
\hline & & & & & $\begin{array}{l}\text { ITS/LSU/ } \\
605\end{array}$ & $\beta T$ & EF-1a & CAL \\
\hline \multirow[t]{2}{*}{1} & \multirow[t]{2}{*}{$\begin{array}{l}\text { Ophiostoma } \\
\text { genhense }\end{array}$} & CFCC 52675 (CXY 2001) T & Larix gmelinii & $\begin{array}{l}\text { Genhe, Inner } \\
\text { Mongolia }\end{array}$ & MK748199 & MN896053 & MN896074 & MN896102 \\
\hline & & CFCC 52676 (CXY 2002) & L. gmelinii & $\begin{array}{l}\text { Genhe, Inner } \\
\text { Mongolia }\end{array}$ & N/A & MN896052 & MN896073 & MN896101 \\
\hline \multirow[t]{5}{*}{2} & \multirow[t]{5}{*}{ O. hongxingense } & CFCC 52695 (CXY 2021) T & L. gmelinii & $\begin{array}{l}\text { Harbin, } \\
\text { Heilongjiang }\end{array}$ & MK748194 & MN896026 & MN896068 & MN896089 \\
\hline & & CFCC 52696 (CXY 2022) & L. gmelinii & $\begin{array}{l}\text { Harbin, } \\
\text { Heilongjiang }\end{array}$ & N/A & MN896028 & MN896067 & MN896090 \\
\hline & & CXY 1905 & L. gmelinii & $\begin{array}{l}\text { Harbin, } \\
\text { Heilongjiang }\end{array}$ & N/A & MN896027 & MN896066 & MN896087 \\
\hline & & CXY 1906 & L. gmelinii & $\begin{array}{l}\text { Harbin, } \\
\text { Heilongjiang }\end{array}$ & N/A & MN896030 & MN896070 & MN896088 \\
\hline & & CXY 1907 & L. gmelinii & $\begin{array}{l}\text { Harbin, } \\
\text { Heilongjiang }\end{array}$ & N/A & MN896029 & MN896069 & MN896091 \\
\hline \multirow[t]{2}{*}{3} & \multirow[t]{2}{*}{ O. lotiforme } & $\begin{array}{l}\text { CFCC } 52691(C X Y 2017= \\
\text { MUCL 55165) T }\end{array}$ & $\begin{array}{l}\text { Pinus sylvestris var. } \\
\text { mongolica }\end{array}$ & $\begin{array}{l}\text { Hailar, Inner } \\
\text { Mongolia }\end{array}$ & MK748185 & MN896036 & $\mathrm{N} / \mathrm{A}$ & N/A \\
\hline & & CFCC 52692 (CXY 2018) & $\begin{array}{l}\text { P. sylvestris var. } \\
\text { mongolica }\end{array}$ & $\begin{array}{l}\text { Hailar, Inner } \\
\text { Mongolia }\end{array}$ & MK748201 & MN896037 & $\mathrm{N} / \mathrm{A}$ & N/A \\
\hline \multirow[t]{2}{*}{4} & \multirow[t]{2}{*}{ O. minus } & CFCC 52697 (CXY 2023) & L. gmelinii & $\begin{array}{l}\text { Arongqi, Inner } \\
\text { Mongolia }\end{array}$ & MK748202 & MN896044 & N/A & N/A \\
\hline & & $\begin{array}{l}\text { CFCC } 52698 \text { (CXY } 2024= \\
\text { MUCL 55157) }\end{array}$ & $\begin{array}{l}\text { P. sylvestris var. } \\
\text { mongolica }\end{array}$ & $\begin{array}{l}\text { Hailar, Inner } \\
\text { Mongolia }\end{array}$ & MK748187 & MN896045 & N/A & N/A \\
\hline \multirow[t]{2}{*}{5} & \multirow[t]{2}{*}{ O. multisynnematum } & CFCC 52677 (CXY 2003) T & L. gmelinii & $\begin{array}{l}\text { Genhe, Inner } \\
\text { Mongolia }\end{array}$ & MK748196 & MN896050 & MN896071 & MN896103 \\
\hline & & CFCC 52678 (CXY 2004) & L. gmelinii & $\begin{array}{l}\text { Genhe, Inner } \\
\text { Mongolia }\end{array}$ & N/A & MN896051 & MN896072 & MN896104 \\
\hline \multirow[t]{4}{*}{6} & \multirow[t]{4}{*}{ O. olgensis } & CFCC 52699 (CXY 2025) & L. gmelinii & $\begin{array}{l}\text { Genhe, Inner } \\
\text { Mongolia }\end{array}$ & MK748204 & MN896048 & N/A & N/A \\
\hline & & CFCC 52700 (CXY 2026) & L. gmelinii & $\begin{array}{l}\text { Genhe, Inner } \\
\text { Mongolia }\end{array}$ & MK748195 & MN896049 & N/A & N/A \\
\hline & & CXY 1908 & L. gmelinii & $\begin{array}{l}\text { Harbin, } \\
\text { Heilongjiang }\end{array}$ & MK748203 & MN896046 & N/A & N/A \\
\hline & & CXY 1909 & L. gmelinii & $\begin{array}{l}\text { Yichun, } \\
\text { Heilongjiang }\end{array}$ & MK748205 & MN896047 & N/A & N/A \\
\hline \multirow[t]{3}{*}{7} & \multirow[t]{3}{*}{ O. peniculi } & CFCC 52687 (CXY 2013) T & L. gmelinii & $\begin{array}{l}\text { Genhe, Inner } \\
\text { Mongolia }\end{array}$ & MK748198 & MN896034 & MN896063 & MN896086 \\
\hline & & CFCC 52688 (CXY 2014) & L. gmelinii & $\begin{array}{l}\text { Genhe, Inner } \\
\text { Mongolia }\end{array}$ & N/A & MN896033 & MN896061 & MN896084 \\
\hline & & CXY 1904 & L. gmelinii & $\begin{array}{l}\text { Genhe, Inner } \\
\text { Mongolia }\end{array}$ & N/A & MN896035 & MN896062 & MN896085 \\
\hline \multirow[t]{6}{*}{8} & \multirow[t]{6}{*}{ O. pseudobicolor } & CFCC 52683 (CXY 2009) T & L. gmelinii & $\begin{array}{l}\text { Genhe, Inner } \\
\text { Mongolia }\end{array}$ & MK748188 & MN896038 & $\mathrm{N} / \mathrm{A}$ & $\mathrm{N} / \mathrm{A}$ \\
\hline & & CFCC 52684 (CXY 2010) & L. gmelinii & $\begin{array}{l}\text { Genhe, Inner } \\
\text { Mongolia }\end{array}$ & MK748190 & MN896040 & N/A & N/A \\
\hline & & $\begin{array}{l}\text { CFCC } 52685(C X Y 2011= \\
\text { MUCL 55168) }\end{array}$ & $\begin{array}{l}\text { L. principis- } \\
\text { rupprechtii }\end{array}$ & $\begin{array}{l}\text { Chifeng, Inner } \\
\text { Mongolia }\end{array}$ & MK748191 & MN896043 & N/A & N/A \\
\hline & & $\begin{array}{l}\text { CFCC } 52686(C X Y 2012= \\
\text { MUCL 55174) }\end{array}$ & L. gmelinii & $\begin{array}{l}\text { Mohe, } \\
\text { Heilongjiang }\end{array}$ & MK748193 & MN896041 & N/A & $\mathrm{N} / \mathrm{A}$ \\
\hline & & CXY 1910 & $\begin{array}{l}\text { L. principis- } \\
\text { rupprechtii }\end{array}$ & $\begin{array}{l}\text { Chifeng, Inner } \\
\text { Mongolia }\end{array}$ & MK748192 & MN896039 & $\mathrm{N} / \mathrm{A}$ & N/A \\
\hline & & CXY 1911 (MUCL 55170) & L. gmelinii & $\begin{array}{l}\text { Tahe, } \\
\text { Heilongjiang }\end{array}$ & MK748189 & MN896042 & $\mathrm{N} / \mathrm{A}$ & N/A \\
\hline
\end{tabular}


Table. 1 Strains of ophiostomatoid fungi sequenced and used for morphological and phylogenetic analysis in this study (Continued)

\begin{tabular}{|c|c|c|c|c|c|c|c|c|}
\hline \multirow[t]{2}{*}{ Taxon } & \multirow[t]{2}{*}{ Species $^{1}$} & \multirow[t]{2}{*}{ Strain no. ${ }^{2.3 .4}$} & \multirow[t]{2}{*}{ Host } & \multirow[t]{2}{*}{ Locality } & \multicolumn{4}{|c|}{ GenBank number ${ }^{5}$} \\
\hline & & & & & $\begin{array}{l}\text { ITS/LSU/ } \\
605\end{array}$ & $\beta T$ & $E F-1 a$ & $C A L$ \\
\hline \multirow[t]{2}{*}{9} & O. rufum & CFCC 52681 (CXY 2007) & L. gmelinii & $\begin{array}{l}\text { Genhe, Inner } \\
\text { Mongolia }\end{array}$ & MK748197 & MN896058 & MN896075 & MN896095 \\
\hline & & CFCC 52682 (CXY 2008) & L. gmelinii & $\begin{array}{l}\text { Genhe, Inner } \\
\text { Mongolia }\end{array}$ & N/A & MN896057 & MN896076 & MN896098 \\
\hline \multirow[t]{2}{*}{10} & O. subelongati & CFCC 52693 (CXY 2019) T & L. gmelinii & $\begin{array}{l}\text { Harbin, } \\
\text { Heilongjiang }\end{array}$ & MK748200 & MN896031 & MN896064 & MN896092 \\
\hline & & CFCC 52694 (CXY 2020) & L. gmelinii & $\begin{array}{l}\text { Harbin, } \\
\text { Heilongjiang }\end{array}$ & N/A & MN896032 & MN896065 & MN896093 \\
\hline \multirow[t]{5}{*}{11} & O. xinganense & CFCC 52679 (CXY 2005) T & L. gmelinii & $\begin{array}{l}\text { Genhe, Inner } \\
\text { Mongolia }\end{array}$ & MK748186 & MN896055 & MN896078 & MN896097 \\
\hline & & CFCC 52680 (CXY 2006) & L. gmelinii & $\begin{array}{l}\text { Genhe, Inner } \\
\text { Mongolia }\end{array}$ & N/A & MN896054 & MN896079 & MN896094 \\
\hline & & CXY 1901 & L. gmelinii & $\begin{array}{l}\text { Genhe, Inner } \\
\text { Mongolia }\end{array}$ & N/A & MN896056 & MN896077 & MN896096 \\
\hline & & CXY 1902 & L. gmelinii & $\begin{array}{l}\text { Genhe, Inner } \\
\text { Mongolia }\end{array}$ & N/A & MN896059 & MN896080 & MN896099 \\
\hline & & CXY 1903 & L. gmelinii & $\begin{array}{l}\text { Genhe, Inner } \\
\text { Mongolia }\end{array}$ & N/A & MN896060 & MN896081 & MN896100 \\
\hline \multirow[t]{2}{*}{12} & $\begin{array}{l}\text { Ceratocystiopsis cf. } \\
\text { pallidobrunnea }\end{array}$ & CFCC 52689 (CXY 2015) & $\begin{array}{l}\text { P. sylvestris var. } \\
\text { mongolica }\end{array}$ & $\begin{array}{l}\text { Hailar, Inner } \\
\text { Mongolia }\end{array}$ & MN892641 & N/A & N/A & N/A \\
\hline & & CFCC 52690 (CXY 2016) & $\begin{array}{l}\text { P. sylvestris var. } \\
\text { mongolica }\end{array}$ & $\begin{array}{l}\text { Hailar, Inner } \\
\text { Mongolia }\end{array}$ & MN892642 & N/A & N/A & N/A \\
\hline \multirow[t]{2}{*}{13} & Leptographium zhangii & CFCC 52701 (CXY 2027) & L. gmelinii & $\begin{array}{l}\text { Genhe, Inner } \\
\text { Mongolia }\end{array}$ & N/A & N/A & MN896082 & N/A \\
\hline & & CFCC 52702 (CXY 2028) & L. gmelinii & $\begin{array}{l}\text { Genhe, Inner } \\
\text { Mongolia }\end{array}$ & N/A & N/A & MN896083 & N/A \\
\hline \multirow[t]{2}{*}{14} & $\begin{array}{l}\text { Endoconidiophora } \\
\text { fujiensis }\end{array}$ & CXY 1912 & L. gmelinii & $\begin{array}{l}\text { Yichun, } \\
\text { Heilongjiang }\end{array}$ & MN896105 & N/A & N/A & N/A \\
\hline & & CXY 1913 & L. gmelinii & $\begin{array}{l}\text { Yichun, } \\
\text { Heilongjiang }\end{array}$ & MN896106 & N/A & N/A & N/A \\
\hline
\end{tabular}

1. Species named in black bold are novel species in this study

2. CFCC China Forestry Culture Collection Center, Beijing, China

3. $C X Y$ the culture collection of the Chinese Academy of Forestry

4. $T$ = ex-holotype isolate

5. ITS Internal transcribed spacer regions 1 and 2 of the nuclear ribosomal DNA operon, including the 5.8S region, LSU Large subunit of the nrDNA, 60S partial 60S ribosomal protein RPL10 gene, $\beta T$ the $\beta$-tubulin gene region, $E F-1 a$ the transcription elongation factor-1a gene region, $C A L$ the calmodulin gene region 